\title{
MicroRNA-181d is a tumor suppressor in human esophageal squamous cell carcinoma inversely regulating Derlin-1
}

\author{
DEJUN LI ${ }^{1}$, MO SHI ${ }^{2}$, HONGSHENG JI ${ }^{1}$, GANG CHEN $^{2}$, HUA JIANG $^{2}$ and ZHOU WANG ${ }^{1,2}$ \\ ${ }^{1}$ Surgical Intensive Care Unit and ${ }^{2}$ Department of Thoracic Surgery, Shandong Provincial Hospital \\ Affiliated to Shandong University, Shandong University, Jinan, Shandong 250021, P.R. China
}

Received March 28, 2016; Accepted July 25, 2016

DOI: $10.3892 /$ or.2016.5028

\begin{abstract}
Esophageal squamous cell carcinoma (ESCC) is the predominant subtype of human esophageal cancer in East Asia. In the present study, we explored the tumor suppressive function of microRNA-181d (miR-181d) in ESCC. Quantitative RT-PCR was used to assess gene levels of miR-181d in both ESCC cell lines and clinical samples. Lentiviral transduction was used to overexpress miR-181d in ECA109 and Kyse30 cells. The possible tumor suppressive effects of miR-181d overexpression on ESCC proliferation, migration and cell cycle transition in vitro, and tumorigenicity in vivo were examined. Downstream target gene of miR-181d in ESCC, Derlin-1 (DERL1) was assessed by luciferase assay and qRT-PCR. DERL1 was also force expressed in miR-181d-overexperssed ECA109 and Kyse30 cells to evaluate its reverse effect on miR-181d mediated tumor suppression in ESCC. miR-181d was aberrantly downregulated in both ESCC cell lines and human tumors. Lentivirus-mediated miR-181d overexpression had significant tumor suppressing functions by inhibiting cancer proliferation, migration and arresting cell cycle transition in vitro, as well as inhibiting tumorigenicity in vivo. DERL1 was identified as downstream target gene of miR-181d in ESCC cells. Forced overexpression of DERL1 in ESCC cells was shown to greatly reverse the tumor suppressive effects of miR-181d upregulation on ESCC in vitro proliferation, migration and cell cycle arrest. Out data suggest that miR$181 \mathrm{~d}$ is a tumor suppressor in ESCC inversely regulating its downstream target gene of DERL1.
\end{abstract}

Correspondence to: Dr Zhou Wang, Department of Thoracic Surgery, Shandong Provincial Hospital Affiliated to Shandong University, Shandong University, 324 Jingwu Road, Jinan, Shandong 250021, P.R. China

E-mail: ericwsw@aol.com

Key words: esophageal squamous cell carcinoma, miR-181d, DERL1, downregulation, overexpression

\section{Introduction}

Esophageal carcinoma is the eighth most common type of cancer worldwide, with high incidence rates and poor prognosis among patients in developing countries $(1,2)$. The two most common types of esophageal carcinoma are esophageal squamous cell carcinoma (ESCC) and esophageal adenocarcinoma (EAC), with ESCC being the most dominant subtype of esophageal carcinoma in East Asia and China, possibly due to smoking and eating habits (3). During past decades, though tremendous progress has been made toward targeted chemotherapy, radiotherapy or advanced surgery to improve clinical outcomes, the overall survival rate for patients with ESCC is still very poor, normally below $30 \%$ (4).

MicroRNAs (miRNAs) are small ( 18 to $22 \mathrm{nt}$ long), non-coding RNAs that post-transcriptionally inhibit gene production or induce protein degradation by binding on the complimentary DNA sequence on the $3^{\prime}$ untranslated region (3' UTR) of the targeted genes $(5,6)$. Studies have shown that miRNAs are differentially expressed in both serum and tumor tissues, thus, playing important regulatory roles, acting as either oncogenes or tumor suppressor genes in human esophageal carcinoma (7-9). MicroRNA-181d (miR-181d), belongs to the family of miR-181, which includes miR-181a, miR-181b, miR-181c and miR-181d. miR-181d has been shown to be aberrantly expressed in various types of carcinomas, and to play critical roles in regulating carcinogenesis, cancer metastasis or cancer apoptosis in human cancers. For example, miR-181d had tumor suppressive effect on glioma migration, apoptosis and cell cycle transition through the regulation of K-ras (10). In addition, miR-181d was shown to be a reliable biomarker for human glioblastoma, and acted as a tumor suppressor to inhibit cancer migration by downregulating methylguaninemethyltransferase (11). In human ESCC, miR-181d, along with miR-335, miR-25, miR-7 and miR-495, were closely associated with the patients gross pathologic classification (12). However, the exact gene expression pattern or possible cancer regulatory role of miR-181d, have not been elucidated.

Gene of Derlin-1 (DERL1) is the partner of ATPase complex, and initially found to regulate misfolding proteins at endoplasmic reticulum $(13,14)$. In human cancers, DERL1 has been mostly reported to be an oncogene of regulating carcinoma progression. In colon cancer, DERL1 was upregulated in carcinoma tissues and promoted cancer proliferation (15). 
In pancreatic cancer, DERL1 was overexpressed in tumor cells and direct targeted therapy on tumor growth in animal models (16). In addition, DERL1 was upregulated in nonsmall cell lung cancer, and induced cancer cell progression and migration through the regulation on EGFR signaling pathways $(17,18)$. Yet, the role of DERL1 in human ESCC has not been determined.

In the present study, we first assessed the gene expression level of miR-181d in both ESCC cell lines and clinical samples from ESCC patients. Secondly, we applied lentiviral transduction in ESCC cell lines, ECA109 and Kyse30 to ectopically upregulate miR-181d. Thirdly, we examined the effects of miR-181d overexpression on ESCC proliferation, migration, cell cycle transition in vitro and ESCC tumorigenicity in vivo. Fourthly, we investigated whether DERL1 was the downstream target gene of miR-181d in ESCC. Finally, we overexpressed DERL1 gene in ESCC cells to assess its functions in regulating ESCC growth in association with miR-181d overexpression.

\section{Materials and methods}

Ethics statements. In the present study, all protocols were approved by the Ethics Committee of the Shandong Provincial Hospital Affiliated to Shandong University (Jinan, Shandong, China). All participating subjects provided a written consent. All procedures were conducted in accordance with the Declaration of Helsinki, local and central regulations for good clinical practice in China.

ESCC cell lines and clinical samples. In the present study, human ESCC cell lines, ECA109, Kyse30, Kyse50, Kyse70, Kyse150 and Kyse510 were purchased from the Cell Bank of Type Culture Collection of Chinese Academy of Sciences (Shanghai, China). A control cell line, esophageal epithelial cell line (HET-1A) was purchased from the American Type Culture Collection (ATCC; Manassas, VA, USA). All cells were maintained in RPMI-1640 medium supplemented with $10 \%$ fetal bovine serum (FBS; Thermo Fisher Scientific, Waltham, MA, USA) at $37^{\circ} \mathrm{C}$ with $95 / 5 \%$ of $\mathrm{O}_{2} / \mathrm{CO}_{2}$.

Between March 2012 and March 2016, paired clinical samples, including ESCC tissues (T) and their adjacent normal esophageal epithelial tissues (ANT) were surgically obtained from 16 patients diagnosed with ESCC in the Department of Thoracic Surgery at Shandong Provincial Hospital Affiliated to Shandong University (Jinan, Shandong, China). Once extracted, all clinical samples were immediately snap-frozen in a bath of liquid nitrogen, labelled and stored at $-70^{\circ} \mathrm{C}$ until RNA extraction for quantitative RT-PCR.

RNA extraction and $q R T-P C R$. RNA extraction was carried out using a TRIzol kit (Thermo Fisher Scientific) according to the manufacturer's protocol. After confirming the quality of RNA extract by a NanoDrop-3000 spectrophotometer (ThermoFisherScientific), reverse-transcription wasperformed using a QuantiTect Reverse Transcription kit (Qiagen Inc., Valencia, CA, USA) according to the manufacturer's protocol. Quantitative real-time polymerase chain reaction (qRT-PCR) was carried out on an ABI Prism 7700 Sequence Detection system (Thermo Fisher Scientific). For quantification on
miR-181d expression, a TaqMan miRNA PCR real-time RT-PCR kit (Applied Biosystems, Foster City, CA, USA) was used with U6 snRNA as sampling control. For quantification of DERL1 expression, a Brilliant SYBR-Green II qRT-PCR kit (Strategene, La Jolla, CA, USA) was used with $18 \mathrm{~s}$ as sampling control. Gene levels of miR-181d and DERL1 were calculated as fold changes relative to U6 or 18s levels and then normalized to the relative gene expression in control samples using the $2^{-\Delta \Delta \mathrm{CT}}$ method, where CT represents cycle thresholds.

miR-181d overexpressing assay. The lentivirus including synthetic miR-181d mimic oligonucleotides were purchased from SunBio Biomedical Technology (Nanjing, China) (Lenti-miR181d-M). The control non-specific lentiviral inhibitor vector (Lenti-C) was also purchased from SunBio Biomedical Technology. ESCC cell lines, ECA109 and Kyse30 cells, were transduced with Lenti-C or Lenti-miR181d-M, along with $10 \mu \mathrm{g} / \mathrm{ml}$ Polybrene, at multiplicity of infection of 20-35. $48 \mathrm{~h}$ later, culture medium was changed to fresh one without lentiviruses and cells were maintained for another 3-5 days to stabilize lentiviral transduction. Transduction efficiency was then confirmed by qRT-PCR.

ESCC in vitro proliferation assay. The in vitro cancer cell growth was assessed by a proliferation assay. Briefly, ECA109 and Kyse 30 cells were plated at 1,000 cells/well in single-cell layer into a 96-well plate. A Vybrant ${ }^{\circledR}$ MTT Cell Proliferation Assay kit (Thermo Fisher Scientific) was then carried out for five consecutive days according to the manufacturer's protocol. Each $24 \mathrm{~h}$, MTT $(5 \mu \mathrm{g} / \mathrm{ml})$ was added to each well for $4 \mathrm{~h}$. Proliferation rates were characterized as recorded optical absorbance at $490 \mathrm{~nm}$ using a V-5000 spectrophotometer reader (Yuanxi Instrument Co., Ltd., Shanghai, China).

ESCC in vitro migration assay. The in vitro migrating capability of ESCC cells was assessed by a Transwell migration assay (Thermo Fisher Scientific) according to the manufacturer's protocol. Briefly, the inserts of Transwell were pre-coated with $0.1 \%$ gelatin (StemCell, USA), then, filled with 5,000 cells/well of ECA109 or Kyse30 cells in culture medium without serum. The lower chambers of Transwell were filled with culture medium with $15 \%$ FBS as migration inducer. After $48 \mathrm{~h}$, the migrated cells in lower chambers were fixed by $70 \%$ ethanol, immune-stained by crystal violet, and photographed using an Olympus IX51 inverted microscopy system (Olympus, Tokyo, Japan).

ESCC in vitro cell cycle assay. The in vitro cell cycle transition of ESCC was assessed by multicolor flow cytometry. Briefly, ECA109 and Kyse30 cells were fixed in ice-cold 70\% ethanol for $10 \mathrm{~min}$, then treated with DNA binding dyes propidium iodide $(20 \mu \mathrm{g} / \mathrm{ml}$; Thermo Fisher Scientific) for $30 \mathrm{~min}$ at $37^{\circ} \mathrm{C}$. Cell DNAs were analyzed using an Epics XL-MCL flow cytometer (Beckman Coulter, Krefeld, Germany) according to the manufacturer's protocol. Cell percentiles at G0/G1, S or M/G2 cycling stages were determined by cytometry histograms using a MultiCycle AV DNA analysis software (Phoenix Flow Systems, San Diego, CA, USA) according to the manufacturer's protocol. 

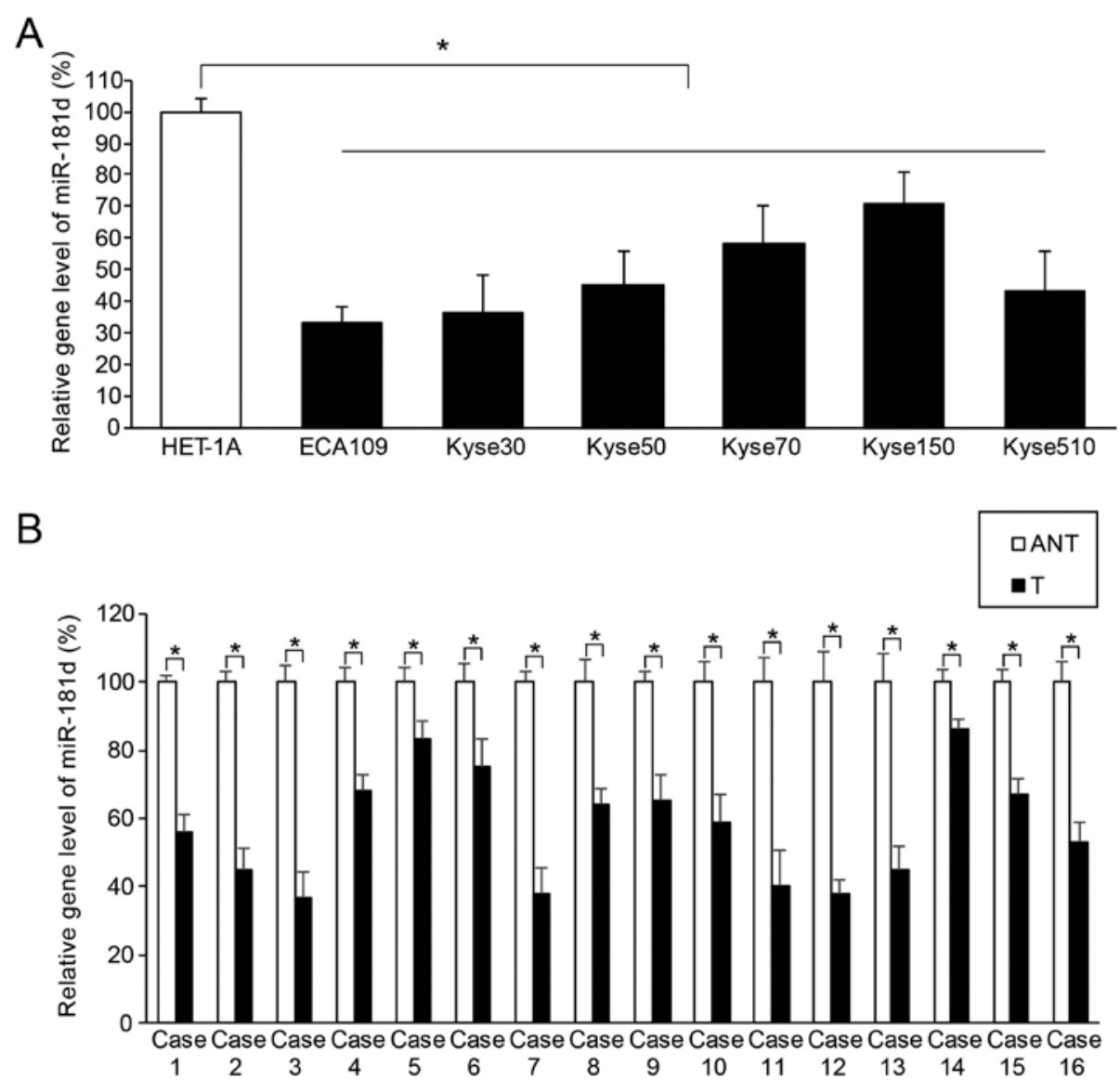

Figure 1. Gene expression pattern of miR-181 in ESCC. (A) Endogenous gene levels of miR-181d were assessed by qRT-PCR, and compared to the control

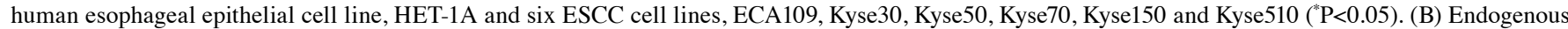
gene levels of miR-181d were also compared between paired clinical samples of ESCC tissues (T) and adjacent normal esophageal epithelial tissues (ANT) in 16 patients with ESCC $\left({ }^{*} \mathrm{P}<0.05\right)$.

ESCC in vivo tumorigenicity assay. The growth of ESCC transplantation was assessed by an in vivo tumorigenicity assay. Briefly, ECA109 cells were subcutaneously injected into abdominal flanks of 6-week-old female athymic nude mice ( 1 million cells/injection, $n=7$ ). The left flanks were injected with cells transduced with Lenti-C, and the right flanks were injected with cells transduced with Lenti-miR181d-M. For five consecutive weeks, in vivo ESCC transplantations were weekly monitored by measuring their sizes $\left(\mathrm{mm}^{3}, l \times w \times w / 2\right.$, where $l$ represents length and $w$ represents width).

Dual-luciferase reporter assay. Human DERL1 3' UTR with putative hsa-miR-181d binding sequences was cloned into a PmirGLO dual-luciferase expression plasmid (Promega, Madison, WI, USA) to create DERL1 luciferase vector. A mutant DERL1 3' UTR without hsa-miR-181d binding sequences was synthesized by SunBio Biomedical Technology, and cloned into PmirGLO to created Mu-DERL1 luciferase vector. HEK293 cells were co-transfected with DERL1 or Mu-DERL1, along with Lenti-C or Lenti-miR181d-M using DharmaFECT4 (Dharmacon) for 48 h. A Dual-Luciferase reporter assay (Promega) was then carried out according to the manufacturer's protocol.

DERL1 overexpressing assay. Full sequence of human DERL1 gene was amplified from a human cDNA library then inserted into a recombinant eukaryotic expression plasmid pcDNA3.1 to create DERL1 overexpressing vector (pcDNA/DERL1). An empty expression plasmid, pcDNA/+, was used as control vector. ECA109 and Kyse30 cells were transfected with pcDNA/+ or pcDNA/DERL1 using Lipofectamine 2000 (Thermo Fisher Scientific) for $48 \mathrm{~h}$. Transfection efficiency was then confirmed by qRT-PCR.

Statistical analysis. All experiments were carried out in triplicates and the data are shown as mean \pm standard errors. Statistical analyses were performed using unpaired Student's t-test on SPSS software (SPSS, Inc., Chicago, IL, USA). Statistical significance was assigned at P-value of $<0.05$.

\section{Results}

miR-181d is aberrantly downregulated in both ESCC cell lines and clinical samples. The gene level of hsa-miR-181d in ESCC was quantitatively assessed using method of qRT-PCR. We found that, in the six human ESCC cell lines, ECA109, Kyse30, Kyse50, Kyse70, Kyse150 and Kyse510, endogenous miR-181d expression was significantly lower than the expression level in the control human esophageal epithelial cell line, HET-1A (Fig. 1A; P<0.05). We also found that, in clinical samples extracted from 16 cases of ESCC patients, miR-181d expression in ESCC tissues (T) was significantly lower than 


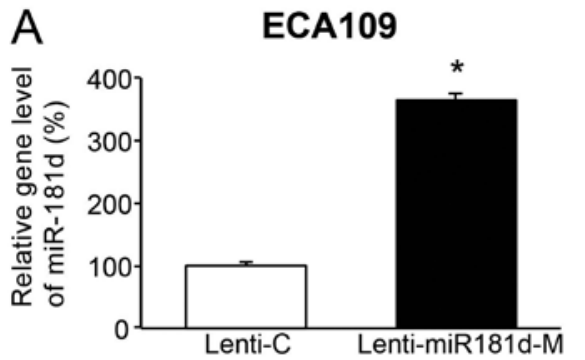

B

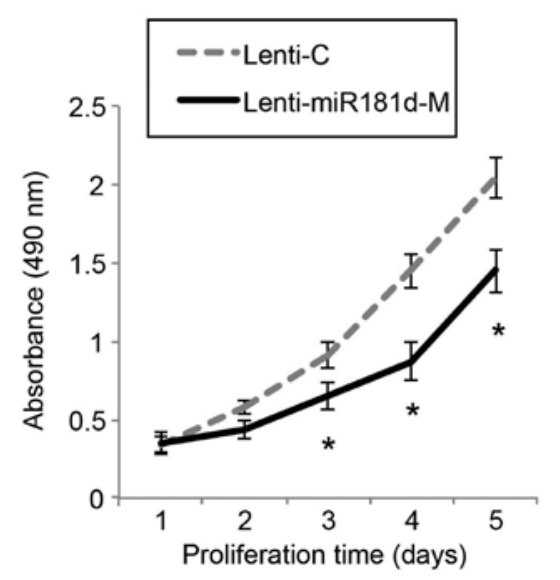

KYSE30

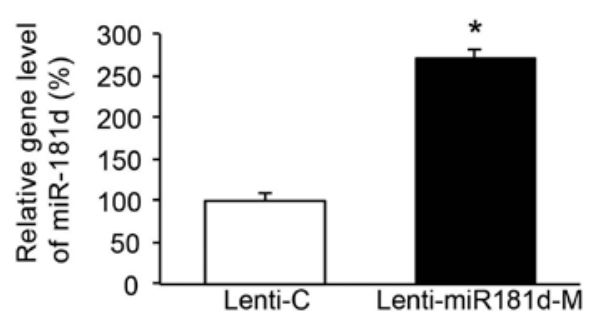

KYSE30

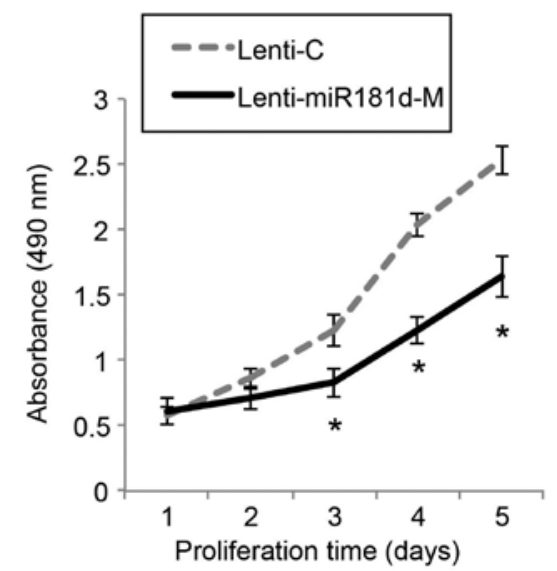

Figure 2. Role of miR-181d overexpression in regulating ESCC in vitro proliferation. (A) ESCC cell lines, ECA109 and Kyse30 cells were transduced with a lentivirus of hsa-181d mimics (Lenti-miR181d-M) or a control lentivirus (Lenti-C). qRT-PCR was used to confirm the overexpression efficiency of lentiviral transduction $\left({ }^{*} \mathrm{P}<0.05\right)$. (B) ECA109 and Kyse30 cells with stable lentiviral-expression were re-plated in 96-well plates. An in vitro proliferation assay was carried out for five consecutive days to compare cancer growth in ESCC cells transduced with Lenti-C and cells transduced with Lenti-miR181d-M. Each $24 \mathrm{~h}$, MTT solution was added in each well and proliferation rates in ECA109 and Kyse30 cells were assessed by measuring the optical absorbance at $490 \mathrm{~nm}$ $\left({ }^{*} \mathrm{P}<0.05\right)$.

miR-181d expression in their adjacent normal esophageal epithelial tissues (ANT) (Fig. 1B; P<0.05). Thus, our data suggest that human miR-181d was aberrantly downregulated in both ESCC cell lines and ESCC tumors in patients.

miR-181d overexpression inhibits ESCC proliferation in vitro. We transduced the ESCC cell lines, ECA109 and Kyse30 cells with Lenti-C or Lenti-miR181d-M to assess the mechanistic regulation of miR-181d overexpression. Five or seven days after lentiviral transduction, qRT-PCR confirmed transduction efficiency by showing that endogenous gene level of miR-181d was significantly upregulated in ESCC cells transduced with Lenti-miR181d-M compared to in cells transduced with Lenti-C (Fig. 2A; P<0.05). The ECA109 and Kyse30 cells were re-suspended and re-seeded in 96-well plates. Cancer growth was then assessed by an in vitro ESCC proliferation assay for five consecutive days. We found in vitro cancer proliferation was greatly inhibited in ESCC cells transduced with LentimiR181d-M than in cells transduced with Lenti-C (Fig. 2B; $\mathrm{P}<0.05)$. Thus, our data suggest that miR-181d is acting as a tumor suppressor in ESCC by inhibiting cancer growth in vitro.

miR-181d overexpression inhibits ESCC migration and cell cycle transition in vitro. We then assessed the effect of miR-181d overexpression on ESCC migration and cell cycle transition in vitro. ECA109 and Kyse30 cells with lentiviral transduction were re-suspended and re-seeded in 96-well plates. An in vitro migration assay was performed. We found that significantly less ECA109 or Kyse30 cells migrated into Transwell lower chambers while they were transduced with Lenti-miR181d-M, than when they were transduced with Lenti-C (Fig. 3A). We also performed a cell cycle assay by multicolor flow cytometry. It showed that significant amount of ESCC cells were arrested at G0/G1 stage while they were transduced with Lenti-miR181d-M, compared when they were transduced with Lenti-C (Fig. 3B; $\mathrm{P}<0.05$ ). Thus, our data suggest that miR-181d overexpression also has tumor suppressive effect on ESCC migration and cell cycle transition in vitro.

miR-181d overexpression inhibits ESCC growth in vivo. We then assessed the effect of miR-181d overexpression on the in vivo growth of ESCC tumors. ECA109 cells with lentiviral transduction were subcutaneously injected into the abdominal regions of female null mice. ECA109 cells with Lenti-C transduction were injected on the left flanks and ECA109 cells with Lenti-miR181d-M transduction on the right flanks. For five consecutive weeks, an in vivo tumorigenicity assay was carried out by comparing the tumor sizes. The results showed that in vivo tumor growth was significantly inhibited in explants transduced with Lenti-miR181d-M than explants transduced with Lenti-C (Fig. 4; P<0.05). Therefore, our data suggest that miR-181d overexpression also had tumor suppressive effect on inhibiting the in vivo proliferation of ESCC tumors. 
A
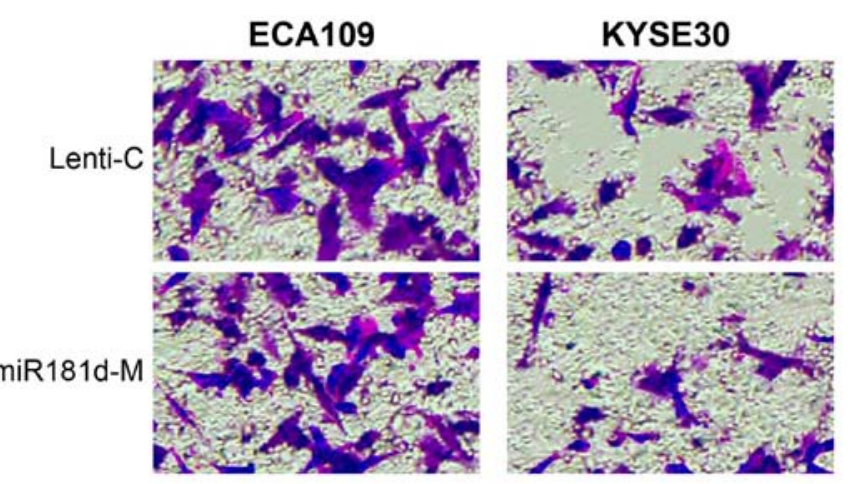

B

ECA109

KYSE30
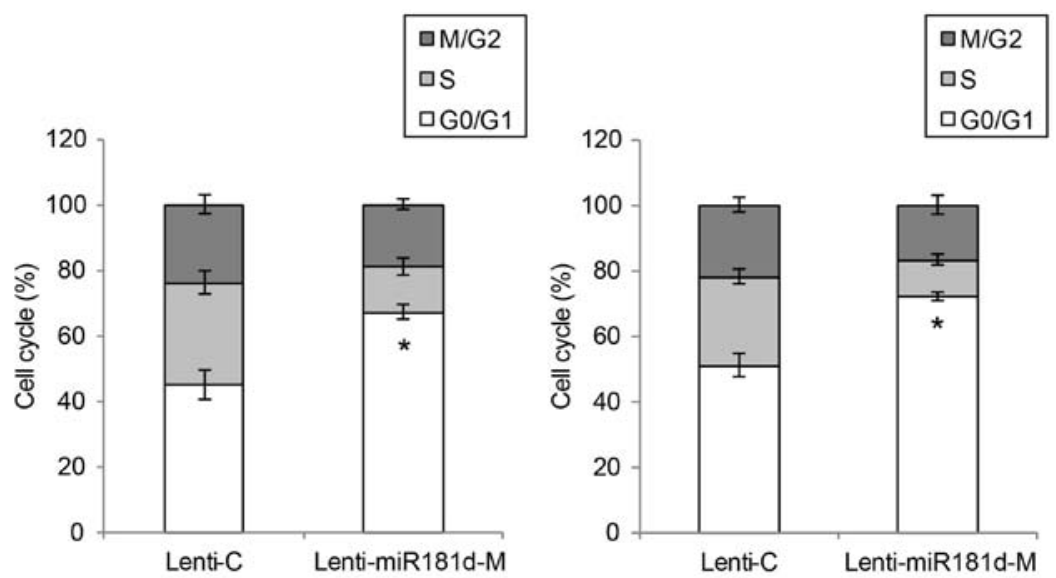

Figure 3. Role of miR-181d overexpression in regulating ESCC in vitro migration and cell cycle transition. (A) In an in vitro Transwell migration assay, ECA109 and Kyse 30 cells with stable lentiviral-expression were plated in the inserts without serum supplement. The lower chambers of the Transwells were filled with medium with 15\% FBS. Forty-eight hours later, migrated cells in the lower chambers were fixed, stained with crystal violet and photographed. Representative images were shown for ECA109 and Kyse30 cells transduced with Lenti-C and those transduced with Lenti-miR181d-M. (B) ECA109 and Kyse30 cells with stable lentiviral-expression were stained with DNA binding dyes, propidium iodide and examined by a multicolor flow cytometry. Relative percentiles of ESCC cells at cell cycle stages of G0/G1, S and GM/G2 were then calculated and compared between ECA109 and Kyse30 cells transduced with Lenti-C and those transduced with Lenti-miR181d-M ("P<0.05).

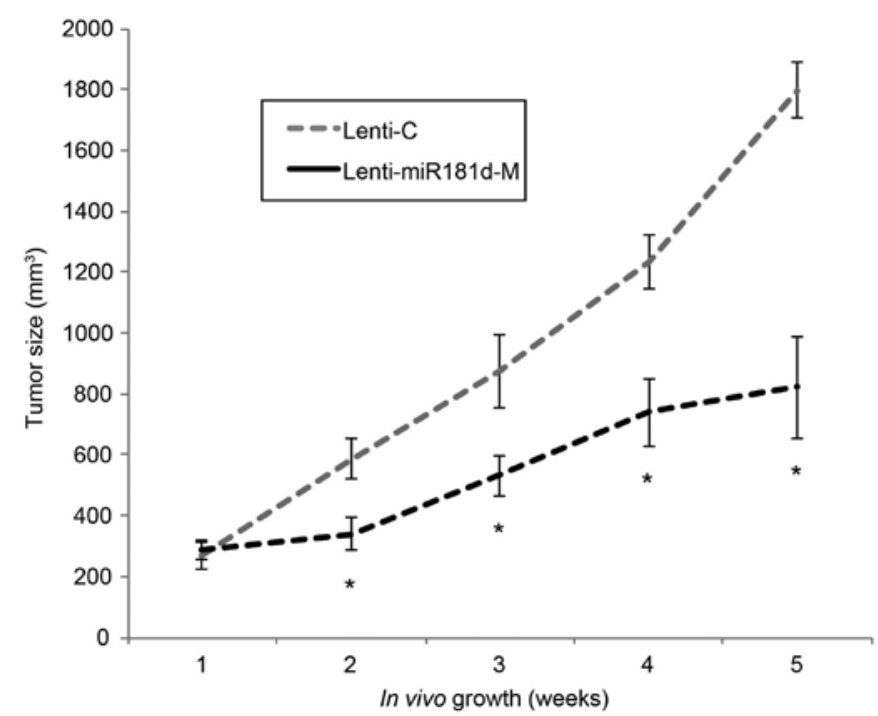

Figure 4. Role of miR-181d overexpression in regulating ESCC in vivo proliferation. In an in vivo tumorigenicity assay, ECA109 cells transduced with Lenti-C were subcutaneously injected in the left abdominal regions of 6-week-old female athymic nude mice. ECA109 cells transduced with Lenti-miR181d-M were injected into right flanks. In vivo tumor sizes were calculated weekly by formula of $l \times w \times w / 2$ ( $l$ represents length and $w$ represents width), and compared between tumor explants transduced with Lenti-C and tumor explants transdued with Lenti-miR181d-M ( $\left.{ }^{*} \mathrm{P}<0.05\right)$.
miR-181d directly regulates downstream target gene of DERL1 in ESCC. We further investigated the downstream molecular targets of miR-181d in ESCC. We found that human DERL1 gene was a candidate gene with a putative miR-181d binding sequence on its 3' UTR (Fig. 5A). To verify this hypothesis, we carried out a dual-luciferase assay. PmirGLO luciferase plasmids containing a wild-type DERL1 3' UTR (DERL1, consisting of miR-181d binding sequence), or a mutated DERL1 3' UTR (mu-DERL1, without miR-181d binding sequence) were co-transfected with Lenti-C or Lenti-miR181d-M in HEK293 cells. Forty-eight hours later, we used a dual-luciferase reporter assay to compare the relative luciferase activities between HEK293 cells transfected with Lenti-C and HEK293 cells transfected with Lenti-miR181d-M. The result showed that relative luciferase activities were significantly different with DERL1 co-transfection, than Mu-DERL1 co-transfection (Fig. 5B; $\mathrm{P}<0.05$ ), confirming that human DERL1 gene is the downstream target of miR-181d.

We also assessed the effect of miR-181d overexpression on gene regulation of DERL1 in ESCC. Analysis of qRT-PCR demonstrated that DERL1 expression was markedly downregulated in ESCC cells transduced with miR-181d-M than in ESCC cells transduced with Lenti-C (Fig. 5C; $\mathrm{P}<0.05$ ). 

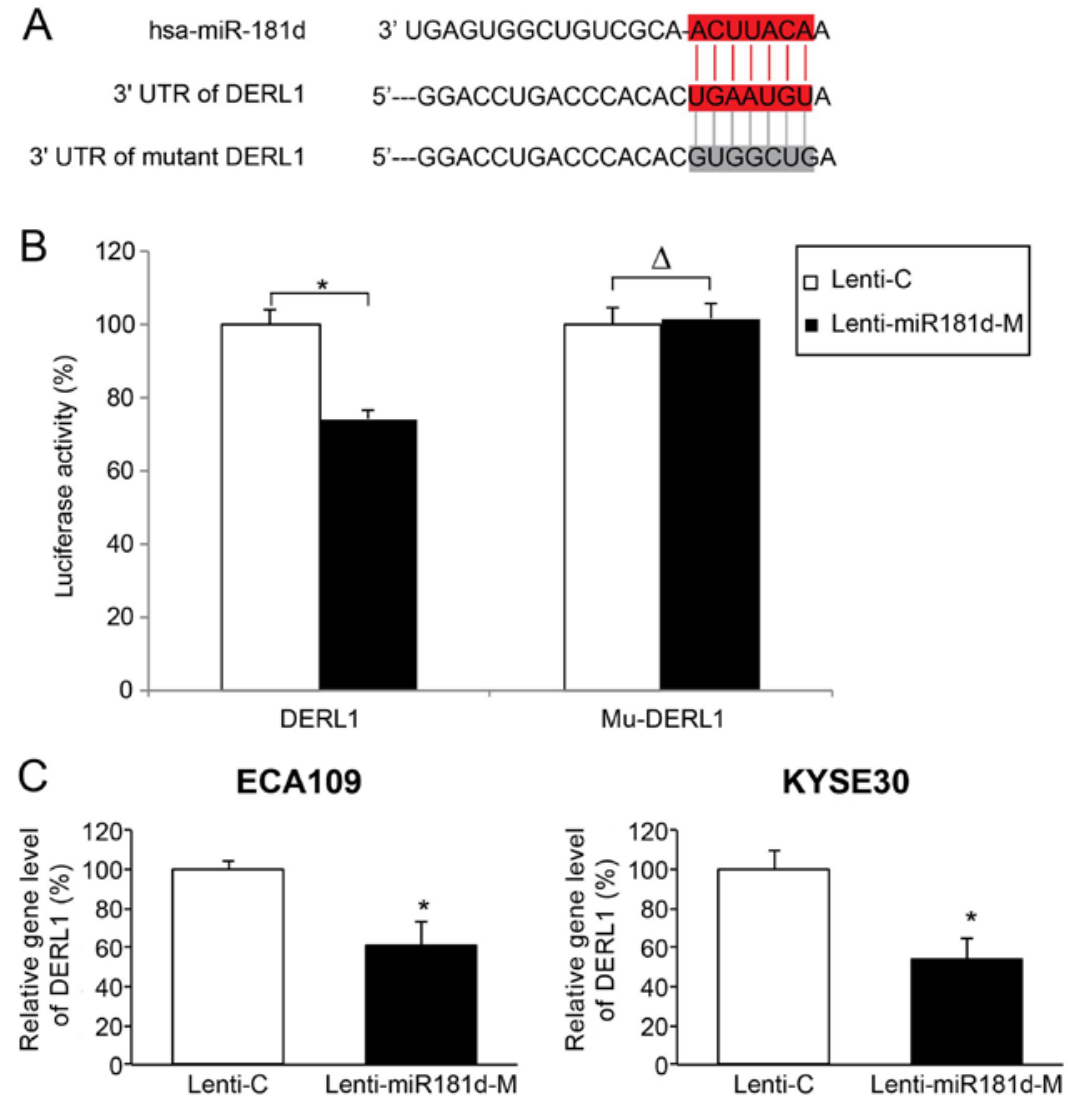

Figure 5. miR-181d directly targets DERL1 gene in ESCC. (A) DNA sequence is shown for putative hsa-miR-181d binding site on 3' UTR of human DERL1 gene. DNA sequence was point-mutated to generate a mutant DERL1 3' UTR without hsa-miR-181d binding site. (B) HEK293 cells were co-transfected with PmirGLO vectors containing a wild-type DERL1 3' UTR, or a mutant DERL1 3' UTR (mu-DERL1), along with Lenti-C or Lenti-miR181d-M. Forty-eight hours later, a dual-luciferase reporter assay was performed $($ ( $P<0.05)$. (C) Gene levels of DERL1 were compared, by qRT-PCR, between ECA109 and Kyse30 cells transduced with Lenti-C and those cells transduced with Lenti-miR181d-M ( $\mathrm{P}<0.05)$.

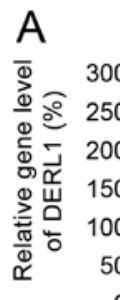

B

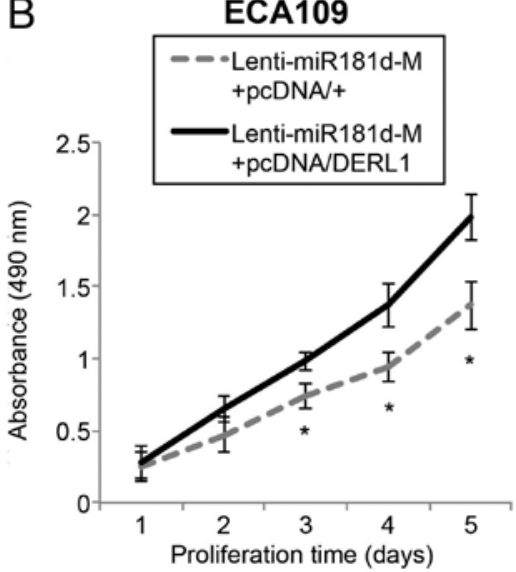

ECA109

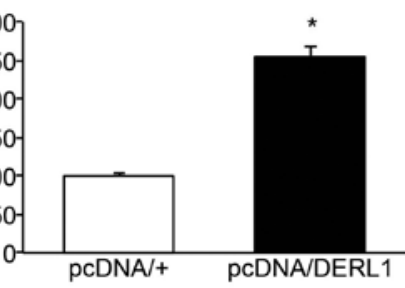

ECA109

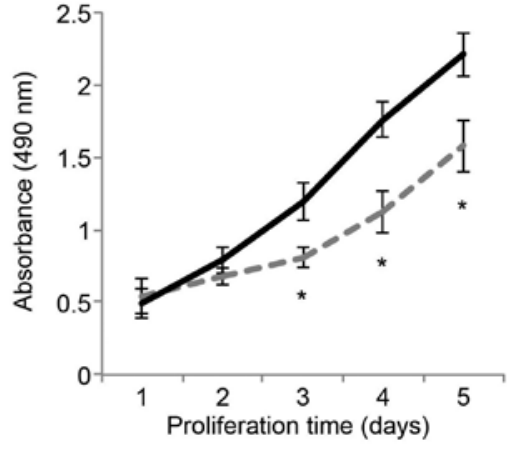

Figure 6. Role of DERL1 upregulation on ESCC proliferation mediated by miR-181d overexpression. (A) ECA109 and Kyse30 cells, transduced with miR181d-M, were further transfected with pcDNA/+ or pcDNA/DERL1. Forty-eight hours later, transfection efficiency was verified by qRT-PCR ("P<0.05), (B) Double-transfected ECA109 and Kyse30 cells were re-plated in 96-well plates. An in vitro proliferation assay was carried out for five days to compare cancer growth in ESCC cells transfected with pcDNA/+ and the cells transfected with pcDNA/DERL1 ("P<0.05). 
A

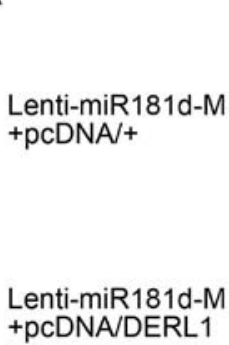

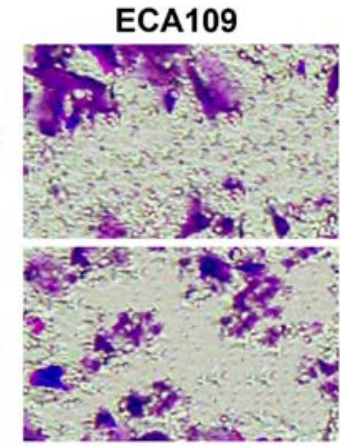

B

ECA109
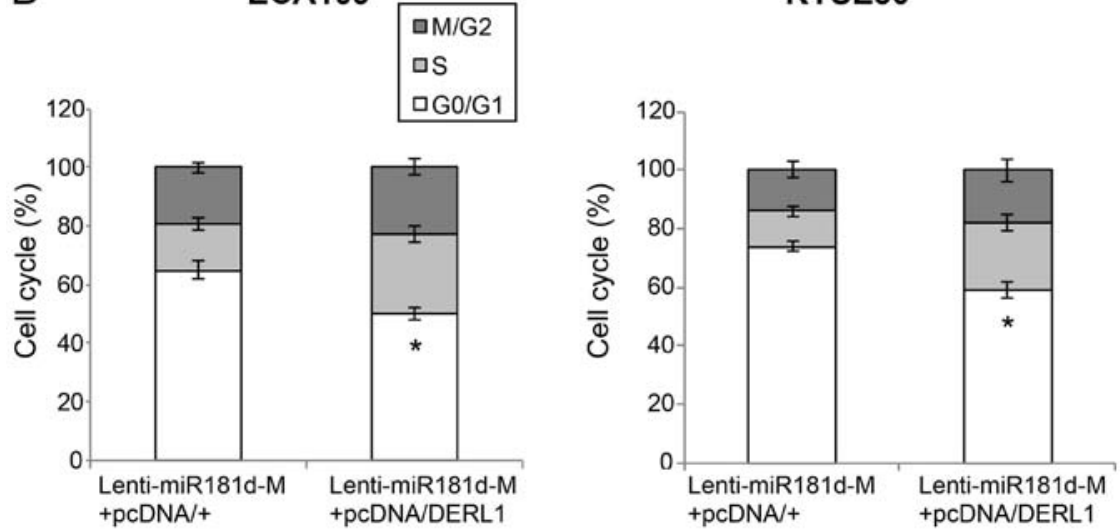

Figure 7. Role of DERL1 upregulation on ESCC migration and cell cycle transition mediated by miR-181d overexpression. (A) In an in vitro Transwell migration assay, double-transfected ECA109 and Kyse30 cells were plated in the inserts without serum supplement. The lower chambers of the Transwells were filled with medium with $15 \%$ FBS. Forty-eight hours later, migrated cells in the lower chambers were fixed, stained with crystal violet and photographed. Representative images are shown for miR-181d-overexpressed ECA109 and Kyse30 cells transfected with pcDNA/+ and those transfected with pcDNA/DERL1. (B) In a cell cycle assay, double-transfected ECA109 and Kyse30 cells were stained with propidium iodide and examined by a multicolor flow cytometry. Relative percentiles of ESCC cells at cell cycle stages of G0/G1, S and GM/G2 were then calculated and compared to miR-181d-overexpressed ECA109 and Kyse30 cells transfected with pcDNA/+ and those cells transfected with pcDNA/DERL1 ( $\left.{ }^{*} \mathrm{P}<0.05\right)$.

Therefore, our data indicate that DERL1 gene was directly modulated by miR-181d in ESCC.

DERL1 upregulation reverses tumor-suppressing effect of miR-181d overexpression in ESCC. Finally, we investigated whether DERL1 was involved in tumor suppression of miR-181d overexpression in ESCC. To achieve this goal, we re-seeded ECA109 and Kyse30 cells with Lenti-miR181d transduction, and then transfected them with an DERL1 overexpressing vector, pcDNA/DERL1 or an empty overexpressing vector, $\mathrm{pcDNA} /+$. Analysis of qRT-PCR verified that, endogenous DERL1 gene level was significantly upregulated by pcDNA/DERL1, rather than pcDNA/+ in miR-181d overexpressed ESCC cells (Fig. 6A; P<0.05).

The double-transfected ECA109 and Kyse30 cells (those transduced with Lenti-miR181d-M, then transfected with pcDNA/+ or pcDNA/DERL1), were re-suspended and re-plated in 96-well plates and assessed with an in vitro proliferation assay. It showed that, in ESCC cells with miR-181d overexpression, DERL1 upregulation greatly promoted in vitro cancer proliferation (Fig. 6B; $\mathrm{P}<0.05$ ).

The double-transfected ECA109 and Kyse30 cells were also examined through the in vitro migration assay. It showed that, in ESCC cells with miR-181d overexpression, DERL1 upregulation markedly induced more cells to migrate into the lower chambers (Fig. 7A). Finally, the in vitro cell cycle assay revealed that cells arrested at G0/G1 stages were markedly relived by DERL1 upregulation (Fig. 7B; $\mathrm{P}<0.05$ ).

Thus, the data of our double-transfection assays suggest that DERL1 upregulation could reverse the tumor suppressive effects of miR-181d overexpression on ESCC proliferation, migration and cell cycle transition.

\section{Discussion}

The expression pattern of miR-181d varies among different types of cancer. In human glioma and glioblastoma, miR-181d was reported to be downregulated in cancer tissues $(10,11)$. However, in human colorectal and gastric cancer, upregulation of miR-181d was seen in tumor tissues $(19,20)$. In human esophageal squamous cell carcinoma (ESCC), though miR181d was reported to be linked to pathological features of cancerous tissue (12), its exact expression pattern was largely unknown. In the present study, we first applied qRT-PCR to investigate the gene expression pattern of miR-181d between ESCC cell lines and control esophageal epithelial cell line, as well as between paired ESCC tumor tissues and adjacent non-cancerous esophageal tissues in human patients. We found that miR-181d was significantly downregulated in both ESCC cell lines and ESCC tumor tissues. Thus, the results of the present study provided strong evidence showing an aberrant expression pattern of miR-181d in ESCC, suggesting that 
miR-181d could be associated with tumor suppressive regulations in ESCC.

We then assessed the functional role of miR-181d in regulating cancer development in ESCC. Lentiviral transduction was shown to successfully upregulate miR-181d expression in ESCC cells ECA109 and Kyse30. The subsequent functional experiments revealed that miR-181d was actually acting as a tumor suppressor in ESCC as it inhibited cancer proliferation, migration, cell cycle transition in vitro and tumor growth in vivo. These data are in line with similar tumor suppressive role of miR-181d previously reported in glioma and glioblastoma, showing miR-181d inhibited cancer proliferation, induced cell cycle arrest and cancer apoptosis in glioma (10), as well as induced temozolomide sensitivity in glioblastoma cells (11). It is worth noting that, though several other studies reported contrary expression pattern, i.e. upregulation of miR-181d in colorectal caner or gastric cancer, no oncogenic functions of miR-181d were revealed in those cancer types $(19,20)$. Therefore, it is very likely that, although the expression of miR-181d may exhibit different patterns, either upregulated or downregulated in different cancers, the regulatory function of miR-181d may be predominantly tumor suppressive, rather than oncogenic.

In addition, in the present study, we explored the molecular signaling pathway associated with tumor suppressive effect of miR-181d in ESCC. Dual-luciferase reporter showed that DERL1 was the downstream target gene of miR-181d. Subsequent qRT-PCR analysis confirmed that DERL1 was inversely downregulated by miR-181d overexpression in ESCC cells. Moreover, experiments of double-transfection by upregulating DERL1 in miR-181d-overexpressed ESCC cells revealed that DERL1 reversed the tumor suppression of miR-181d overexpression on ESCC proliferation, migration and cell cycle transition. DERL1 was often found to be overexpressed in human cancers, including colon (15), lung (17) or pancreatic cancer (16). Thus, our data are in line with previous studies, showing that DERL1 could be an active oncogenic gene in various types of human cancers, including ESCC.

In conclusion, in the present study, we provided strong evidence showing tumor suppressive role of miR-181d in human ESCC. The molecular mechanism of miR-181d in ESCC progression may very likely act through the inverse regulation of the oncogene DERL1.

\section{References}

1. Herszényi L and Tulassay Z: Epidemiology of gastrointestinal and liver tumors. Eur Rev Med Pharmacol Sci 14: 249-258, 2010.

2. Napier KJ, Scheerer M and Misra S: Esophageal cancer: A Review of epidemiology, pathogenesis, staging workup and treatment modalities. World J Gastrointest Oncol 6: 112-120, 2014.
3. Zhang HZ, Jin GF and Shen HB: Epidemiologic differences in esophageal cancer between Asian and Western populations. Chin J Cancer 31: 281-286, 2012.

4. Liu J, Xie X, Zhou C, Peng S, Rao D and Fu J: Which factors are associated with actual 5-year survival of oesophageal squamous cell carcinoma? Eur J Cardiothorac Surg 41: e7-e11, 2012.

5. O'Malley MA, Elliott KC and Burian RM: From genetic to genomic regulation: Iterativity in microRNA research. Stud Hist Philos Biol Biomed Sci 41: 407-417, 2010.

6. Guarnieri DJ and DiLeone RJ: MicroRNAs: A new class of gene regulators. Ann Med 40: 197-208, 2008.

7. Liu SG, Qin XG, Zhao BS, Qi B, Yao WJ, Wang TY, Li HC and Wu XN: Differential expression of miRNAs in esophageal cancer tissue. Oncol Lett 5: 1639-1642, 2013.

8. Gu J, Wang Y and Wu X: MicroRNA in the pathogenesis and prognosis of esophageal cancer. Curr Pharm Des 19: 1292-1300, 2013.

9. Wu C, Wang C, Guan X, Liu Y, Li D, Zhou X, Zhang Y, Chen X, Wang J, Zen K, et al: Diagnostic and prognostic implications of a serum miRNA panel in oesophageal squamous cell carcinoma. PLoS One 9: e92292, 2014.

10. Wang XF, Shi ZM, Wang XR, Cao L, Wang YY, Zhang JX, Yin Y, Luo H, Kang CS, Liu N, et al: MiR-181d acts as a tumor suppressor in glioma by targeting K-ras and Bcl-2. J Cancer Res Clin Oncol 138: 573-584, 2012.

11. Zhang W, Zhang J, Hoadley K, Kushwaha D, Ramakrishnan V, Li S, Kang C, You Y, Jiang C, Song SW, et al: miR-181d: A predictive glioblastoma biomarker that downregulates MGMT expression. Neuro-oncol 14: 712-719, 2012.

12. Guo Y, Chen Z, Zhang L, Zhou F, Shi S, Feng X, Li B, Meng X, Ma X, Luo M, et al: Distinctive microRNA profiles relating to patient survival in esophageal squamous cell carcinoma. Cancer Res 68: 26-33, 2008.

13. Wang F, Olson EM and Shyng SL: Role of Derlin-1 protein in proteostasis regulation of ATP-sensitive potassium channels. J Biol Chem 287: 10482-10493, 2012.

14. Chen SF, Wu CH, Lee YM, Tam K, Tsai YC, Liou JY and Shyue SK: Caveolin-1 interacts with Derlin-1 and promotes ubiquitination and degradation of cyclooxygenase-2 via collaboration with p97 complex. J Biol Chem 288: 33462-33469, 2013.

15. Tan X, He X, Jiang Z, Wang X, Ma L, Liu L, Wang X, Fan Z and Su D: Derlin-1 is overexpressed in human colon cancer and promotes cancer cell proliferation. Mol Cell Biochem 408: 205-213, 2015 .

16. Ran Y, Hu H, Hu D, Zhou Z, Sun Y, Yu L, Sun L, Pan J, Liu J, Liu T, et al: Derlin-1 is overexpressed on the tumor cell surface and enables antibody-mediated tumor targeting therapy. Clin Cancer Res 14: 6538-6545, 2008.

17. Dong QZ, Wang Y, Tang ZP, Fu L, Li QC, Wang ED and Wang EH: Derlin-1 is overexpressed in non-small cell lung cancer and promotes cancer cell invasion via EGFR-ERK-mediated up-regulation of MMP-2 and MMP-9. Am J Pathol 182: 954-964, 2013.

18. Xu L, Wang ZH, Xu D, Lin G, Li DR, Wan T and Guo SL: Expression of Derlin-1 and its effect on expression of autophagy marker genes under endoplasmic reticulum stress in lung cancer cells. Cancer Cell Int 14: 50, 2014.

19. Motoyama K, Inoue H, Takatsuno Y, Tanaka F, Mimori K, Uetake H, Sugihara K and Mori M: Over- and under-expressed microRNAs in human colorectal cancer. Int J Oncol 34: 1069-1075, 2009.

20. Ueda T, Volinia S, Okumura H, Shimizu M, Taccioli C, Rossi S, Alder H, Liu CG, Oue N, Yasui W, et al: Relation between microRNA expression and progression and prognosis of gastric cancer: A microRNA expression analysis. Lancet Oncol 11: 136-146, 2010. 